This manuscript is a preprint and has been submitted for publication in Geophysics. Please note that, despite having undergone peer-review, the manuscript has yet to be formally accepted for publication. Subsequent versions of this manuscript may have slightly different content. If accepted, the final version of this manuscript will be available via the 'Peer-reviewed Publication DOI' link on the right-hand side of this webpage. Please feel free to contact any of the authors; we welcome feedback. 


\title{
Training a physics-guided convolutional neural network for seismic inversion
}

\author{
Jian Sun ${ }^{12}$, Kristopher A. Innanen ${ }^{3}$, Chao Huang ${ }^{4}$ \\ ${ }^{1}$ Dept. of Geoscience, The Pennsylvania State University, University Park, PA, USA; \\ ${ }^{3}$ Dept. of Geoscience, University of Calgary, Calgary, AB, Canada; ${ }^{4}$ School of Ocean and \\ Earth Science, Tongji University, Shanghai, China
}

(July 13, 2020)

Running head: Physics-guided deep learning inversion

\begin{abstract}
The determination of subsurface elastic property models is crucial in quantitative seismic data processing and interpretation. This problem is commonly solved by deterministic physical methods, such as tomography or full waveform inversion (FWI). However, these methods are entirely local, and require accurate initial models. Deep learning represents a plausible class of methods for seismic inversion, which may avoid some of the issues of purely descent-based approaches; it has a growing record of providing solutions for general image processing tasks. However, any generic deep learning network capable of relating each elastic property cell value to each sample in a seismic dataset would require a very large number of degrees of freedom. Two approaches might be taken to train such a network. First, by invoking a massive and exhaustive training dataset; second, by working to reduce the degrees of freedom by enforcing physical constraints on model-data relationship. The second approach is referred to as "theory-guiding". Based on recent progress of wave theory-designed network, we propose a hybrid network design, involving both de-
\end{abstract}


terministic, physics-based modelling and data-driven deep learning components. From an optimization standpoint, both a data-driven model misfit (i.e., standard deep learning), and now a model-driven data misfit (i.e., a wave propagation network), are simultaneously minimized during the training of the network. We compare the prediction capability of this physics-guided network with that of a fully data-driven counterpart in a synthetic salt velocity model building problem. The results indicate that the fully data-driven neural network acts like a smooth global inversion method: it recovers a large amount of large scale structural information, including the location and approximate boundaries of salt bodies, and background trends, directly from raw seismograms. The physics guided component appears to act to enhance detail and to more highly resolve structures, including salt body boundaries, and interfaces of background layers. 


\section{INTRODUCTION}

Estimation of subsurface structures and physical parameters using seismic data can be carried out based on fully deterministic models of wave propagation. This category of approach includes, for instance, traditional tomography (Woodward et al., 2008) for velocity model building by migrating seismograms with traveltime information, and full waveform inversion (FWI) for the high resolution reconstruction of subsurface velocity, or elastic property models (Tarantola, 1984; Virieux and Operto, 2009). These approaches are powerful, and in particular in the case of the latter, represent the state of the art in seismic inversion. However, they tend to be computationally complex, with results and computational requirements that are both very sensitive to parameterization, data completeness, initial model, and numerical optimization approach.

Deep learning, a recent extension of machine learning technology, has drawn significant recent attention across various fields, including seismology. Deep learning applications to problems of image processing include classification (Krizhevsky et al., 2012; Simonyan and Zisserman, 2014), segmentation(Long et al., 2015; Ronneberger et al., 2015; Häggström et al., 2019), denoising (Zhang et al., 2017), and superresolution (Kim et al., 2016). They are often carried out with convolutional neural networks (CNNs), a popular class of network which is unusual in that it can be formulated with a relatively small number of trainable parameters. For example, Simonyan and Zisserman (2014) observed unusually high performance in a CNN called VGG16, which included a convolutional encoder and a classifier, for image recognition; Ronneberger et al. (2015) designed a CNN with an encoder and decoder architecture in an U-shape (Unet), and reported high accuracy in its application to biomedical image segmentation tasks. 
Approaches of this general type have been applied successfully in geophysical disciplines also. However, here we encounter challenges of the kind discussed by Karpatne et al. (2018), because we tend to have (1) large and rich, but not exhaustive, datasets, and (2) accurate physical models, in the form of partial differential equations, which relate data to subsurface properties. In the relatively rare circumstance in which exhaustive data are available for a given geophysical problem, successes are striking. For instance, Wu et al. (2019) demonstrated that a CNN can be trained to predict the presence of faults, as well as their strikes and dips; Ovcharenko et al. (2019) applied low-frequency extrapolation from multi-offset seismic data using a CNN; Kaur et al. (2019) formulated elastic wave mode-separation in heterogeneous anisotropic media using a generative adversarial network (GAN); Yang and Ma (2019) set up a velocity building procedure for subsurface salt bodies using an Unet, and discussed the influence of low-frequencies and noise. These approaches are fully data driven, and thus rely entirely on the completeness of training data. Supplying such data could be difficult or impossible for the solution of a range of important geophysical problems, to the point where they would seem to be essentially inaccessible by a deep learning approach. For instance, if a FWI-like problem was broached, in which each sample of a large seismic data set must be mapped to each element of a multidimensional elastic property model, the number of degrees of freedom within the deep learning network would be exceedingly large. Training data of the extent needed to constrain such a network are unlikely to be available.

However, these issues, which derive from item (1) above, can be addressed to a great degree by making use of item (2). That is, the degrees of freedom of an otherwise vast and complex network can be reduced by constraining the network with physical rules. For 
instance, Wu and McMechan (2019) showed that an FWI procedure can be set up within a CNN framework with a multi-grid parameterization of the subsurface model, using a multilayer neural network. Meanwhile, Sun et al. (2020) and Richardson (2018) discussed a theory-guided waveform inversion using a recurrent neural network (RNN) designed to emulate wave propagation; the former authors further confirmed that the training stage within such a waveform RNN is equivalent to the optimization of standard, gradient-based FWI. Zhang et al. (2020) extended this to incorporate more complete, multidimensional elastic and viscoelastic, models and parameter estimations. These networks, as initially realized, were sufficiently well-constrained by the rules of acoustic or elastic wave propagation, that they were successfully trained with a single training data set (hence their resemblance to standard FWI). However, ideally, such networks would allow for a greater role for training when additional data exist, and to date that has not been broached in the literature. Here, we examine the opportunity deep learning networks offer for hybridizing data-driven training with waveform inversion, by constructing a convolutional neural network in which both data-driven model misfits and physics-based data residuals are simultaneously minimized.

This paper is organized as follows. First, we review the deep learning formulation of seismic inverse problems, and then describe the architecture of our proposed network. Second, we examine the response of a fully data-driven (i.e., non physics-guided) network in a $2 \mathrm{D}$ synthetic salt velocity model building environment, highlight advantages but also disadvantages of this approach. With some of these features having been established, we will re-incorporate the physics-guided component within the same CNN architecture to establish the role the physical constraints play. 


\section{DATA-DRIVEN NETWORK DESIGN FOR SEISMIC INVERSION}

\section{Problem formulation}

Let the seismic inverse problem as solved within a neural network be the determination of the medium property model vector $\mathbf{m}$ from the observed seismic data $\mathbf{d}$ :

$$
\tilde{\mathbf{m}}=\Omega(\mathbf{d} ; \Theta)
$$

where the operator $\Omega(\cdot)$ contains the full nonlinear relationship between the input data and the estimated subsurface property $\tilde{\mathbf{m}}$, and involves a sequence of nonlinear transforms with trainable weights $\Theta$. In a neural network, the nonlinearity is commonly included using activation mapping, such as rectified linear unit, or ReLU (Nair and Hinton, 2010). $\Omega(\cdot)$ is the inverse of a forward operator $\mathcal{F}(\cdot)$ which contains all wave propagation rules as well as source and acquisition information, i.e., $\mathbf{d}=\mathcal{F}(\mathbf{m})$. For a seismic velocity model building problem, the inputs are seismic shot gathers and the outputs are velocity models. The best-fit mapping from input to output is determined in a training stage, during which all trainable parameters $\Theta$ are estimated using a training dataset. This is carried out in a numerical optimization procedure, in which an objective function measuring the model misfit (i.e., the discrepancy between the estimated velocity $\tilde{\mathbf{m}}$ and the ground-truth velocity model $\mathbf{m}$ ) is minimized. In the case of an $\ell 2$-norm, for instance, this objective function is

$$
\mathcal{L}_{m}^{n}(\mathbf{m}, \mathbf{d})=\frac{1}{n} \sum_{i=0}^{n}\left\|\mathbf{m}_{i}-\tilde{\mathbf{m}}_{i}\right\|^{2}=\frac{1}{n} \sum_{i=0}^{n}\left\|\mathbf{m}_{i}-\Omega\left(\mathbf{d}_{i} ; \Theta\right)\right\|^{2}
$$

where the label $m$ is a reminder that the function pertains to model rather than data misfit; $n$ is the number of paired samples in the training data distribution.

To manage the large number of training samples normally involved, and also the mem- 
ory limitations of either graphic processing units (GPU) or tensor processing units (TPU), a mini-batch strategy is applied in both training and validation stages. Rather than minimizing $\mathcal{L}_{m}^{n}$ with $n$ covering the entire training dataset in a single optimization, the training samples are randomly divided into batches, each containing $k$ samples ( $k$ is referred to as the batch size). The weights are calculated by minimizing the objective function over each batch, via:

$$
\hat{\Theta}=\underset{\Theta}{\arg \min } \mathcal{L}_{m}^{k}(\mathbf{m}, \mathbf{d})=\underset{\Theta}{\arg \min } \frac{1}{k} \sum_{i=1}^{k}\left\|\mathbf{m}_{i}-\Omega\left(\mathbf{d}_{i} ; \Theta\right)\right\|^{2} .
$$

A single epoch of training is defined as a single passing of all training batches forward and backward through the neural network. In velocity model building problems, model parameters have a large range of possible values which can be plausibly taken on, and velocity models may be of high and low variance. Elements within the random batches in these situations can make unequal contributions to the objective function, with highvariance velocity models tending to dominate in the convergence process. To balance the contributions of the samples in a single batch, in our general network we adopt a normalized objective function:

$$
\hat{\Theta}=\underset{\Theta}{\arg \min } \tilde{\mathcal{L}}_{m}^{k}(\mathbf{m}, \mathbf{d})=\underset{\Theta}{\arg \min } \frac{1}{k} \sum_{i=1}^{k}\left\|\frac{\mathbf{m}_{i}-\Omega\left(\mathbf{d}_{i} ; \Theta\right)}{\max \left(\mathbf{m}_{i}\right)+\epsilon}\right\|^{2} .
$$

In the training stage of deep neural networks, optimization algorithms are typically gradient-based, and are formulated with or without momentum. In previous work (Sun et al., 2020), we found that the convergence of the seismic velocity inversion problem using the Adaptive Momentum, or Adam, algorithm (Kingma and Ba, 2014), with an appropriate learning rate, was comparable or even better than that of standard adjoint-state methods. Based on this finding, the Adam algorithm is adopted for all optimization processes in this paper. 


\section{Network architecture}

We select an encoder-decoder CNN design. In it, the encoder accepts seismic shot gathers as input and generates low-dimensional feature maps through a sequence of convolution blocks and maxpooling layers. Then, the decoder transforms these feature maps into the estimated velocity model using a sequence of convolution blocks and transposed convolution layers (this latter process is more commonly known as "deconvolution" in the deep learning community, but to avoid confusion with the geophysical term we will continue to refer to it as "transposed convolution"). The architecture of the CNN is plotted in Figure 1. There are four partitions in the proposed network, including an initial convolution, an encoder network, a decoder network, and a sequence of scaling and clamping processes. The initial convolution (plotted as the pink column in Figure 1) contains a single convolutional layer with 16 convolutional filters of size $1 \times 1 \times 10$. Thus the network accepts 10 shot gathers per model in the velocity model building. The purpose of this initial convolution layer is to generate an adaptive input with an appropriate number of channels for the encoder network.

[Figure 1 about here.]

[Figure 2 about here.]

After the initial convolution, the maps are fed into the encoder partition, which is comprised of five convolutional blocks (plotted as the brown columns in Figure 1) and four maxpooling layers (plotted as the dark-blue columns in Figure 1). These convolutional blocks share the same structure, including two convolution layers, two batch normalization 
layers, and two activation layers using a leaky rectifier unit (LeakyReLU), as illustrated in the left panel of Figure 2. In the encoder, the number of output channels $(n c)$ generated by a convolutional block is twice that of its input channels $(c)$, i.e., $n c=2 c$, while the feature map size remains. The sequence of convolutional blocks and maxpooling layers implies that, by passing through the encoder, the dimension of the input is significantly reduced, but the output is richer because of the increased number of channels. The encoder thus transforms the high-dimensional input feature maps into low-dimensional, detailed latent space representations.

Next, these low-dimensional feature maps in latent space are used as input for the decoder, which is a sequential list of four transposed convolution layers (plotted as the darkgreen columns in Figure 1) with four cross-interacting convolution blocks, following by a final convolution combination (plotted as the light-blue column in Figure 1). In the decoder, the output channel from a convolution block is half of the input channel (i.e., $n c=c / / 2$ ), but the same size remains in its other dimensions. The last convolution combination contains a single convolution layer with one filter of size $1 \times 1 \times 32$, followed by a ReLU layer, as illustrated in the right panel of Figure 2. A scaling procedure is then performed to assign the output into to a velocity value, i.e., $\tilde{\mathbf{m}}=\tilde{\mathbf{m}} *\left(\mathbf{m}_{\max }-\mathbf{m}_{\min }\right)+\mathbf{m}_{\min }$, where $\mathbf{m}_{\min }$ and $\mathbf{m}_{\max }$ represent the global minimum and maximum of velocity values. Finally, a clamping step (shown in bottom of Table 1) is required to extract the estimated velocity models. In addition, a constant negative leaking slope 0.1 is applied within all LeakyReLU layers in the architecture. Detailed descriptions of layer parameters are contained in Table 1.

[Table 1 about here.] 


\section{VELOCITY MODEL BUILDING WITH A DATA-DRIVEN CNN}

In this section, we carry out a simulated velocity building exercise for a subsurface salt body using the basic network architecture set out in the previous section. The goal is to create a benchmark deep learning solution using our proposed encoder-decoder CNN in a fully data-driven mode. This will both highlight the potential strengths of a deep learning technique, but also to highlight some practical challenges, and use these to motivate the physics-guided version we introduce in the next section.

\section{Data preparation}

Optimizing the network illustrated in Figure 1 requires a very large number of training samples. Here, 20,000 synthetic velocity models with a grid size of $100 \times 100$ and $10 \mathrm{~m}$ cells are created. Each model contains a single salt body with an arbitrary shape and location. The salt velocity is fixed at $4500 \mathrm{~m} / \mathrm{s}$. The backgrounds are random realizations of 5 -to- 12 laterally-varying intervals with velocity values ranging from $1500-4000 \mathrm{~m} / \mathrm{s}$. These synthetic velocity models are divided into the training (17,000 samples) and validation (3,000 samples)

datasets. In Figure 3 ten example velocity models extracted from the training dataset are illustrated; ten velocity samples selected from the validation dataset are likewise plotted in Figure 4.

[Figure 3 about here.]

[Figure 4 about here.] 
To generate the corresponding shot gathers as inputs of the proposed CNN, we adopt a second-order (both in spatial and time dimensions) finite difference operator for the forward wave propagation simulation. For each velocity model, ten shot gathers are collected with shot interval 100m. All ten sources are located at the surface starting from 50m and ending at $950 \mathrm{~m}$ (from left to right). A minimum phase wavelet with $12 \mathrm{~Hz}$ dominant frequency and a simple absorbing boundary condition are used for the synthetic seismic record collection. The time sample interval during wave propagation is $1.5 \mathrm{~ms}$ and 800 time samples are recorded. However, to reduce memory use and to fit the input size of our designed CNN, we further downsample all shot gathers into 400 time samples with $3 \mathrm{~ms}$ interval. In Figure 5, ten shot gathers related to the upper-left velocity model in Figure 4 are plotted. Boundary reflections are observed in shot gathers, however we judge that these will not strongly affect our conclusions.

[Figure 5 about here.]

The ten raw shot gathers for each velocity model are treated as distinct input channels in the proposed CNN; no preprocessing is applied. To accelerate the convergence process during training, all velocity models are scaled into the range $[0,1]$ using a global minimum and maximum values: $\mathbf{m}=\left(\mathbf{m}-\mathbf{m}_{\min }\right) /\left(\mathbf{m}_{\max }-\mathbf{m}_{\min }\right)$ where $\mathbf{m}_{\min }=1500 \mathrm{~m} / \mathrm{s}$ and $\mathbf{m}_{\min }=4500 \mathrm{~m} / \mathrm{s}$. The model misfit is calculated between the scaled velocity profiles and the output of the decoder network using equation 4. 


\section{Training and validation}

Training of the proposed CNN is carried out on 40 NVIDIA Telsa P100-PCIe GPU accelerators with $16 \mathrm{~GB}$ memory. A batch size of 64 is chosen for training; the validation process is performed with a batch size of 128 after every training epoch. The Adam algorithm with the learning rate 0.001 is used to optimize the CNN. In each epoch, we compute the mean squared error (MSE) over all batches; their average is plotted in Figure 6. The trend indicates that the CNN converges within 200 epochs using a normalized objective function.

Ten velocity models are estimated using the trained data-driven CNN, given raw shot gathers as input. In Figure 7, ten ground-truth velocity models are plotted in the first row, and the associated data-driven CNN predicted velocities are plotted in the second row. Generally, the salt body locations and the velocity values within the bodies are accurately predicted by the data-driven CNN. The salt body boundaries have been reasonably approximated, with the background trends and model character largely intact. It is fair to say that the CNN, trained by the pure model misfit objective function, has the capacity to capture some of the property and positioning information contained in the raw shot gathers. One could, for instance, consider using such data-driven CNN results for rough seismic interpretation or as an initial model for FWI.

However, especially in so far as these examples are synthetic, and so represent something like the best results possible for such a network, the question of how to improve the output (i.e., create more accurate, better resolved, inversions) is an important one. The obvious answer in this case is using increased training samples, but we hold that the size of an exhaustive training data set would be too large for this to be a sensible direction to pursue, 
even in these small examples. Instead, we will move to a deep learning approach which achieves this without inflating the training data set size, by imposing constraints on the form of physical rules on an otherwise similar CNN architecture.

[Figure 6 about here.]

[Figure 7 about here.]

\section{PHYSICS-GUIDING IN NEURAL NETWORK DESIGN}

Sun et al. (2020) demonstrated that in the mapping between velocity and wave data, the physics of wave propagation can be explicitly enforced within a theory-designed RNN. Such a network is optimized by a crosscorrelation, in time, of the backpropagated data residuals and the second-order partial derivative of the forward-modeled states, in a manner equivalent to a gradient-based FWI. Unlike data-driven neural network training, which searches for the best-fit mapping between input and output by adjusting internally weighted connections, the theory-designed RNN optimizes the input by minimizing the data discrepancy in the context of the physical rules. Here, we assemble hybrid network able to learn simultaneously from a data-driven model misfit and a physics-based deterministic data residual.

\section{Wave theory-designed RNN}

Given an estimated set of subsurface model parameters $\tilde{\mathbf{m}}$, the physics-based data residual can be obtained by forward-propagating information through a theory-designed waveform 
RNN operator $\mathcal{F}(\cdot)$. The data residual in an $\ell 2$-norm is in this case

$$
\mathcal{L}_{d}^{k}(\tilde{\mathbf{m}}, \mathbf{d})=\frac{1}{k} \sum_{i=1}^{k} \sum_{s=1}^{n_{s}} \sum_{t=0}^{T}\left\|\mathbf{d}_{i}^{s, t}-\mathcal{F}^{s, t}\left(\tilde{\mathbf{m}}_{i}\right)\right\|^{2}
$$

where $k$ denotes the batch size, $t$ is the time step in the RNN, i.e., seismic sample interval, $n_{s}$ is the number of sources per subsurface model, and $\tilde{\mathbf{d}}_{i}^{s, t}=\mathcal{F}^{s, t}\left(\tilde{\mathbf{m}}_{i}\right)$ represents the waveform RNN forward propagation. Optimal inputs are those subsurface physics parameters which minimize equation 5 . In a normalized form, it is written

$$
\tilde{\mathbf{m}}=\underset{\tilde{\mathbf{m}}}{\arg \min } \tilde{\mathcal{L}}_{d}^{k}(\tilde{\mathbf{m}}, \mathbf{d})=\underset{\tilde{\mathbf{m}}}{\arg \min } \frac{1}{k} \sum_{i=1}^{k} \sum_{s=1}^{n_{s}} \sum_{t=0}^{T}\left\|\frac{\mathbf{d}_{i}^{s, t}-\mathcal{F}^{s, t}\left(\tilde{\mathbf{m}}_{i}\right)}{\max \left(\mathbf{d}_{i}\right)+\epsilon}\right\|^{2}
$$

In gradient-descent training, the model update is based on the gradient of $\mathcal{L}_{d}(\tilde{\mathbf{m}}, \mathbf{d})$ with respect to the model parameters $\tilde{\mathbf{m}}$ :

$$
\delta \tilde{\mathbf{m}}=-\frac{\partial \tilde{\mathcal{L}}_{d}^{k}}{\partial \tilde{\mathbf{m}}}=2 \sum_{i=1}^{k} \sum_{s=1}^{n_{s}} \sum_{t=0}^{T}\left[\frac{\partial \tilde{\mathcal{L}}_{d}^{k}}{\partial \tilde{\mathbf{d}}_{i}^{s, t}}\right] \frac{\partial \tilde{\mathbf{d}}_{i}^{s, t}}{\partial \tilde{\mathbf{m}}}
$$

where $\left[\partial \tilde{\mathcal{L}}_{d}^{k} / \partial \tilde{\mathbf{d}}_{i}^{s, t}\right]$ is determined by the detailed structure of the forward operator $\mathcal{F}(\cdot)$, and contains all partial derivatives via the chain rule. For instance, with the temporal secondorder finite difference operator in Figure $8,\left[\partial \tilde{\mathcal{L}}_{d}^{k} / \partial \tilde{\mathbf{d}}_{i}^{s, t}\right]$ contains three contributions at time steps $t, t+\Delta t, t+2 \Delta t$, respectively:

$$
\left[\frac{\partial \tilde{\mathcal{L}}_{d}^{k}}{\partial \tilde{\mathbf{d}}_{i}^{s, t}}\right]=\left[\frac{\partial \tilde{\mathcal{L}}_{d}^{k}}{\partial \tilde{\mathbf{d}}_{i}^{s, t+2 \Delta t}}\right] \frac{\partial \tilde{\mathbf{d}}^{s, t+2 \Delta t}}{\partial \tilde{\mathbf{d}}_{i}^{s, t}}+\left[\frac{\partial \tilde{\mathcal{L}}_{d}^{k}}{\partial \tilde{\mathbf{d}}_{i}^{s, t+\Delta t}}\right] \frac{\partial \tilde{\mathbf{d}}^{s, t+\Delta t}}{\partial \tilde{\mathbf{d}}_{i}^{s, t}}+\frac{\partial \tilde{\mathcal{L}}_{d}^{k}}{\partial \tilde{\mathbf{d}}_{i}^{s, t}}
$$

where $0 \leq t \leq T, T$ being the maximum time of wave propagation, and where the initial conditions for the RNN backpropagation are assumed to be zeros, i.e., $\left[\partial \tilde{\mathcal{L}}_{d}^{k} / \partial \tilde{\mathbf{d}}_{i}^{s, t}\right]_{t=T+\Delta T, T+2 \Delta T}=$ 0. A detailed derivation of the gradient for a full time sequence (i.e., time steps from 0 to $T$ ) using the chain rule appears elsewhere (Sun et al., 2020).

[Figure 8 about here.] 
The auto-differential backpropagation method for neural networks requires storage of all related internal states, which is computationally expensive, especially for RNN backpropagation. We therefore apply truncated backpropagation instead of the full time sequence backpropagation in equation 6 . For a full time sequence RNN $(0 \rightarrow T)$, initial conditions are applied at time steps $T+1$, and $T+2$ which are initialized as zeros. The truncated approach instead divides the RNN cells into several time segments, such as $0 \rightarrow(l-1) \Delta t$, $l \Delta t \rightarrow(2 l-1) \Delta t, \cdots, t-l \Delta t \rightarrow t, \cdots, n l \Delta t \rightarrow T$, and zero initial conditions are applied at each time breaking steps $l \Delta t,(l+1) \Delta t, 2 l \Delta t,(2 l+1) \Delta t, \cdots, t+\Delta t, t+2 \Delta t, \cdots$, $T+\Delta t, T+2 \Delta t$. For example, considering a time segment $t-l \Delta t$ to $t$ (see Figure 8 ). The backpropagation is performed from $t$ to $t-l \Delta t$. According to equation 8 , the partial derivatives of the data residual at time step $t$ comprise three partitions. However, we lack information at time step $t+\Delta t$ and $t+2 \Delta t$ in a truncated RNN, so these two par-

tial derivative contributions are initialized to zero, i.e., $\left[\partial \tilde{\mathcal{L}}_{d}^{k} / \partial \tilde{\mathbf{d}}_{i}^{s, t}\right]_{t=t+\Delta t, t+2 \Delta t}=0$. The truncated approach is in this sense only approximately equivalent to the full time sequence RNN backpropagation; it does, however, permit a trade-off to be made between the speed of convergence and memory requirements.

\section{A physics-guided network for seismic inversion}

We now analyze a hybrid network, which contains an encoder-decoder CNN of the type set up in the previous section, and illustrated in Figure 1, and a theory-designed waveform RNN, as illustrated in shown in Figure 8. The hybrid network is illustrated in Figure 9. Raw shot gathers are input into the encoder-decoder CNN; the output CNN-estimated subsurface model parameters are then used as inputs for the physics-based deterministic RNN, from 
which we obt ain simulated seismic records. The model misfit is calculated between the CNN estimated results and the ground-truth velocity models, and the data residual is computed between the simulated seismic records and raw shot gathers. This permits an objective function to be formed from a weighted sum of model misfit (equation 4) and data residual (equation 6):

$$
\mathcal{L}^{k}=\tilde{\mathcal{L}}_{m}^{k}+\lambda \tilde{\mathcal{L}}_{d}^{k}
$$

where $\lambda$ moderates trade-off between model and data misfits, respectively; in the experiments to follow, we set $\lambda=10$.

[Figure 9 about here.]

\section{VELOCITY MODEL BUILDING WITH A PHYSICS-GUIDED NEURAL NETWORK}

In this section, the same salt body velocity model building problem is used to examine the training and validation of the physics-guided network which contains both data-driven model misfit and physics-based data residual measures.

\section{Training and validation}

The network with architecture illustrated in Figure 9 is now trained in a physics-guided manner using the same training and validation datasets applied in the data-driven experiment. The trainable parameters only appear in the encoder-decoder CNN component; the parameter settings are those of the fully data-driven CNN illustrated in Figure 1. To manage memory limitations on the GPUs, we reset the batch size for training and validation 
to 16 and 64 respectively. A truncated time length of 50 is chosen for the truncated RNN backpropagation component. The network as a whole is trained with the same computer equipment, and identical training parameters, as those used in the earlier data-driven CNN.

[Figure 10 about here.]

In Figure 10, we plot the model misfit, data misfit, and their weighted summation during the physics-guided training of the network. These plots indicate that the model misfit dominates the optimization process during the first few epochs. Subsequently, the network appears to converge to a local minima of model misfit. Our expectation from the earlier analysis is that this type of model misfit drives towards a solution containing approximate salt body information and basic velocity trends within background layers. In other words, it plays a role similar to initial velocity model building in FWI. This is essential for the subsequent physics-guided convergence process, to the extent that it resembles FWI.

We observe that in the first few epochs the corresponding data misfit is not reduced. There are two main reasons for this. First, the network parameters are randomly initialized, which causes the estimated velocity models at very first few epochs have no physical meaning; second, the model misfit objective function only provides a pixel-wise optimization, and physics constraints are not being invoked. However, after several epochs, the optimization begins to be guided by the physics-based data misfit. During such a convergence process, the model misfit remains while the reduction of the data residual continues, which indicates the starting of an approximate physics-driven optimization stage. We point out that, although the model misfit dominated starting point acts something like an initial velocity model building stage, and the data-misfit ending point in isolation acts something like an 
FWI regimen, the mid-range iterations, in which both act simultaneously, is unique to this hybrid approach.

The output of the new network is illustrated in Figure 11. In it, ten estimated velocity models extracted from the validation dataset are plotted. Compared to the results derived from the fully data-driven CNN (in Figure 7), the salt bodies predicted here have more highly resolved shapes, and clearly defined boundaries, reduced smoothing. The results also exhibit better definition within background irregular layers, and in facts resolves their interfaces, something which was not achieved with our data-driven CNN. In the first and second rows of Figure 12 the estimated model misfits using both the data-driven and physicsguided networks respectively are plotted. We observe that the physics-guided objective function reduces the risks of producing artificial anomalies, such as those apparent in the panel fourth from the right. In Figure 13, the data residuals from middle shot gathers are also plotted, alongside the final estimated velocity models from both the data-driven and the physics-guided networks. Again, we observe reduced discrepancies.

In Figure 14, ten additional velocity models are extracted from the validation dataset. From top to bottom, the figure includes plots of: the ground-truth velocity, the data-driven estimated results, the physics-guided network results, model misfits from the data-driven CNN, and model misfits from the physics-guided network. In Figure 15 shot gathers from the results in Figure 14 from the first row to the bottom, in that order, are plotted.

[Figure 11 about here.]

[Figure 12 about here.] 
[Figure 13 about here.]

[Figure 14 about here.]

[Figure 15 about here.]

\section{DISCUSSION}

It appears from our analysis that the data-driven $\mathrm{CNN}$ is capable of accurately extracting the main subsurface parameter information influencing the waveforms observed in surface seismic data; its results would appear to be capable, for instance, of providing good starting models for a standard FWI. Physics-guiding appears to be able to supply a significant jump in accuracy and resolution, which we suggest would only be possible in a data-driven scheme through a large inflation of the training data and computational time. Further quantitative comparisons between the data-driven $\mathrm{CNN}$ and the physics guided network is required to completely flesh out the trade-offs between the two elements of the hybrid approach, and how a physics-constrained objective function operates within the optimization of an otherwise "traditional" neural network.

We can begin this process relatively simply. We carry out the velocity building process on the entire validation dataset, which comprises 3000 velocity models, using both the data-driven $\mathrm{CNN}$ and the physics guided networks. After acquiring the estimated velocity models, mean squared model misfits are computed for both. Also, with these estimated velocity models, forward wave propagation is performed to calculate the mean squared data residuals. The results are plotted in Figure 16, where the blue ellipse shows the 
predicted error associated with the data-driven CNN, and the red ellipse is the predicted error associated with the physics-guided network. The centers of the ellipses represent the mean of model MSE over the entire validation dataset. The heights of the ellipses represent twice the standard deviation of the model MSE; the widths are twice the standard deviation of the data MSE. We observe that the model MSE of the physics-guided results are slightly better than those of the data-driven CNN. However, the data MSE of the physics-guided network results are much smaller compared to those of the the data-driven CNN. The small standard deviation of the data MSE is an indicator of stability and robustness in the physics-guiding approach.

[Figure 16 about here.]

We surmise that involving the physics-based objective function in the training of the neural network, we gain more information from the same training dataset. However, weaknesses of the physics-based approach, familiar to FWI practitioners, such as illumination issues, and susceptibility to missing or incomplete data, are incorporated at the same time. For example, the physics-guided network results struggles to delineate lower boundaries of salt bodies, as is visible in Figure 11; this is a familiar feature in imaging based on wave methods (Liu et al., 2011; Malcolm et al., 2009). Whether increasing the training aspect of a network like the one we discuss could mitigate these shortcomings, and if so how to select for them, are questions of ongoing research. 


\section{CONCLUSIONS}

Deep learning, as a class of artificial intelligence methods, has produced a wide and growing range of solutions for imaging processing problems. However, the accuracy and stability of the underlying supervised neural networks is highly dependent on the scope of the training data, and more practically on the availability of the computational resources required to carry out sufficient training. In large seismic imaging and inversion problems, exhaustive training data sets are largely unavailable. Furthermore, seismic data being the projection of a field, which satisfies to some degree a known partial differential equation, is strongly suggestive that a full data-driven network, trained "from scratch", is not necessary to explain much of the data variance. Therefore, to better employ deep learning techniques in solving geoscience problems, including physics-guiding, and keeping the training data volumes manageably small, is a sensible strategy.

In this paper, we use waveform-based velocity building of salt bodies as a way to enumerate the positive and negative features of a fully data-driven CNN. Our experimentation indicates that, with a particular training data set, a fully data-driven CNN is capable of capturing the main features of subsurface salt body models, and to function in a global manner, meaning without the explicit definition of a nearby starting model. In fact, these results are regarded as being likely candidates for initial models for conventional FWI. However, we also observe a lack of detail and an inability to resolve local salt body boundary and background layer information. The natural route is to explore a significant increase in the training of this CNN; however, recent work in theory-guided machine learning in seismic inversion is suggestive of a second possibility. 
To improve the network while keeping the training data set size and extent unchange, we introduce "physics-guiding", analyzed in isolation in a separate communication, which involves data residuals, and a wave propagation model to explain them, in the training stage. The aim is to ensure that, while "learning", the neural network penalizes updates which are inconsistent with wave propagation within the current iterate of velocity model. Comparing results of this constrained network to those of the data-driven CNN, we observe a strong up-tick in accuracy, and resolution.

\section{ACKNOWLEDGMENTS}

This work was carried out in collaboration with CREWES and as an outgrowth of research funded by CREWES industrial sponsors and NSERC (Natural Science and Engineering Research Council of Canada) through the grant CRDPJ 461179-13. 


\section{REFERENCES}

Häggström, I., C. R. Schmidtlein, G. Campanella, and T. J. Fuchs, 2019, Deeppet: A deep encoder-decoder network for directly solving the pet image reconstruction inverse problem: Medical image analysis, 54, 253-262.

Karpatne, A., I. Ebert-Uphoff, S. Ravela, H. A. Babaie, and V. Kumar, 2018, Machine learning for the geosciences: Challenges and opportunities: IEEE Transactions on Knowledge and Data Engineering.

Kaur, H., S. Fomel, and N. Pham, 2019, Elastic wave-mode separation in heterogeneous anisotropic media using deep learning, in SEG Technical Program Expanded Abstracts 2019, 2654-2658, Society of Exploration Geophysicists.

Kim, J., J. Kwon Lee, and K. Mu Lee, 2016, Accurate image super-resolution using very deep convolutional networks: Proceedings of the IEEE conference on computer vision and pattern recognition, 1646-1654.

Kingma, D. P. and J. Ba, 2014, Adam: A method for stochastic optimization: arXiv preprint arXiv:1412.6980.

Krizhevsky, A., I. Sutskever, and G. E. Hinton, 2012, Imagenet classification with deep convolutional neural networks: Advances in neural information processing systems, 10971105

Liu, Y., X. Chang, D. Jin, R. He, H. Sun, and Y. Zheng, 2011, Reverse time migration of multiples for subsalt imaging: Geophysics, 76, WB209-WB216.

Long, J., E. Shelhamer, and T. Darrell, 2015, Fully convolutional networks for semantic segmentation: Proceedings of the IEEE conference on computer vision and pattern recognition, 3431-3440. 
Malcolm, A. E., B. Ursin, and V. Maarten, 2009, Seismic imaging and illumination with internal multiples: Geophysical Journal International, 176, 847-864.

Nair, V. and G. E. Hinton, 2010, Rectified linear units improve restricted boltzmann machines: Proceedings of the 27th international conference on machine learning (ICML-10), $807-814$

Ovcharenko, O., V. Kazei, M. Kalita, D. Peter, and T. Alkhalifah, 2019, Deep learning for low-frequency extrapolation from multioffset seismic data: Geophysics, 84, R989-R1001.

Richardson, A., 2018, Seismic full-waveform inversion using deep learning tools and techniques: arXiv preprint arXiv:1801.07232.

Ronneberger, O., P. Fischer, and T. Brox, 2015, U-net: Convolutional networks for biomedical image segmentation: International Conference on Medical image computing and computer-assisted intervention, 234-241.

Simonyan, K. and A. Zisserman, 2014, Very deep convolutional networks for large-scale image recognition: arXiv preprint arXiv:1409.1556.

Sun, J., Z. Niu, K. A. Innanen, J. Li, and D. O. Trad, 2020, A theory-guided deep-learning formulation and optimization of seismic waveform inversion: Geophysics, 85, R87-R99.

Tarantola, A., 1984, Inversion of seismic reflection data in the acoustic approximation: Geophysics, 49, 1259-1266.

Virieux, J. and S. Operto, 2009, An overview of full-waveform inversion in exploration geophysics: Geophysics, 74, WCC1-WCC26.

Woodward, M. J., D. Nichols, O. Zdraveva, P. Whitfield, and T. Johns, 2008, A decade of tomography: Geophysics, 73, VE5-VE11.

Wu, X., Y. Shi, S. Fomel, L. Liang, Q. Zhang, and A. Z. Yusifov, 2019, Faultnet3d: pre- 
dicting fault probabilities, strikes, and dips with a single convolutional neural network: IEEE Transactions on Geoscience and Remote Sensing, 57, 9138-9155.

Wu, Y. and G. A. McMechan, 2019, Parametric convolutional neural network-domain fullwaveform inversion: Geophysics, 84, R881-R896.

Yang, F. and J. Ma, 2019, Deep-learning inversion: A next-generation seismic velocity model building method: Geophysics, 84, R583-R599.

Zhang, K., W. Zuo, Y. Chen, D. Meng, and L. Zhang, 2017, Beyond a gaussian denoiser: Residual learning of deep cnn for image denoising: IEEE Transactions on Image Processing, 26, 3142-3155.

Zhang, T., K. Innanen, J. Sun, and D. Trad, 2020, Numerical analysis of a deep learning formulation of elastic full waveform inversion: SEG Technical Program Expanded Abstracts 2020. 


\section{LIST OF FIGURES}

1 The architecture of our proposed encoder-decoder convolution neural network (CNN). The structures of conv_block and conv_last are illustrated in Figure 2 and detailed parameters are contained in Table $1 \ldots \ldots . . . . .$.

2 Left: the detailed structure of convolutional block (conv_block) shown as the brown columns in Figure 1, where $(k, c / n c, h, w)$ denotes (batch size, input/output channel, height, width), respectively. For encoder $n c=2 * c$ and for decoder $n c=c / / 2$. Right: the structure of the last convolutional combination (conv_last) in decoder plotted as the blue column in Figure 1.

3 Ten velocity models extracted from the training dataset. . . . . . . . . . .

4 Ten velocity samples selected from the validation dataset. . . . . . . . . . .

5 One input example containing ten shot gathers using the velocity model shown in the upper-left corner in Figure 4 . . . . . . . . . . . . . . . . . . 31

6 MSE during the training stage using equation 4 as the objective function. .

7 Comparison of the ground-truth (the top row) and the data-driven CNN estimated (the bottom row) velocity models. . . . . . . . . . . .

8 Truncated structure of waveform RNN, where the green (red) arrows delineate the forward (backward) propagation direction. . . . . . . . . . . . .

9 The structure of a physics-guided network involving a data-driven CNN illustrated in Figure 1 and a waveform-based RNN plotted in Figure 8. . . .

10 The misfits behavior during a physics-guided training of the network. a) mean squared model misfit $\left.\tilde{\mathcal{L}}_{m}, b\right)$ mean squared data misfit $\tilde{\mathcal{L}}_{d}, c$ ) weighted summation: $\tilde{\mathcal{L}}_{m}+10 \tilde{\mathcal{L}}_{d} \ldots \ldots \ldots \ldots \ldots \ldots$

11 Comparison of the ground-truth velocity models and the physics-guided network estimated results. . . . . . . . . . . . . . . . . .

12 Model misfit comparison between the data-driven CNN and the physicsguided network. . . . . . . . . . . . . . . . . .

13 Data residual comparison between the data-driven CNN and the physicsguided network. . . . . . . . . . . . . . . . . .

14 Ten additional velocity samples extracted from the validation dataset. From top to bottom: the ground-truth velocity models, the data-driven estimated results, the physics-guided network results, model misfits from the datadriven CNN, and model misfits from the physics-guided network. . . . . . .

15 Ten additional middle-located shot gathers and data residuals associated with velocity models, in the same order, illustrated in Figure 14. . . . . . . . . .

16 Model and data MSEs analysis between the data-driven CNN and the physicsguided network. . . . . . . . . . . . . . . . . 


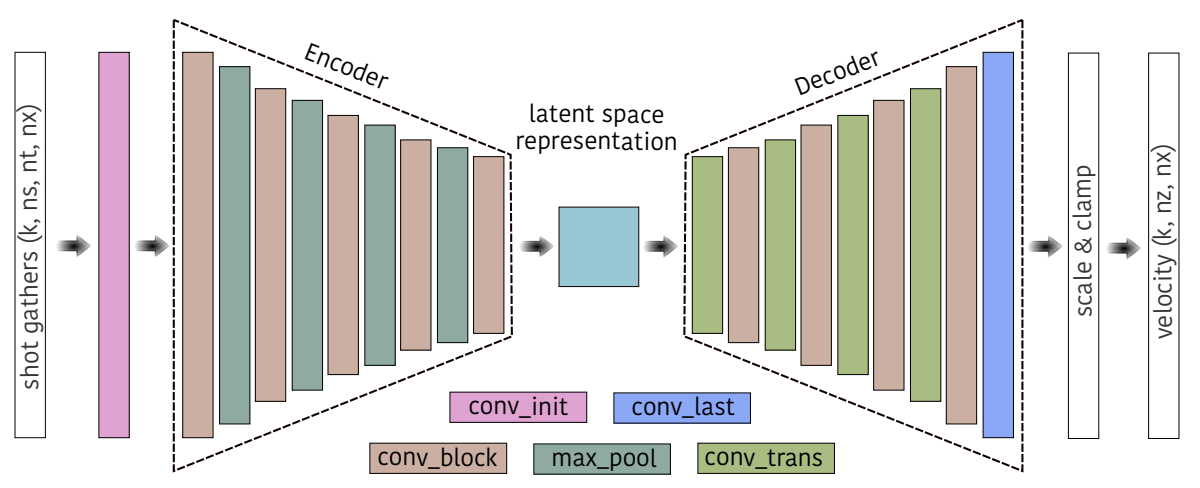

Figure 1: The architecture of our proposed encoder-decoder convolution neural network $(\mathrm{CNN})$. The structures of conv_block and conv_last are illustrated in Figure 2 and detailed parameters are contained in Table 1. 

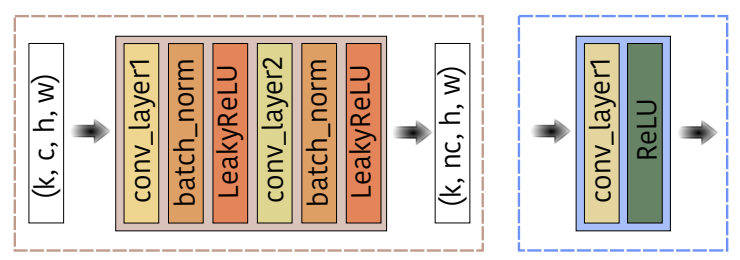

Figure 2: Left: the detailed structure of convolutional block (conv_block) shown as the brown columns in Figure 1, where $(k, c / n c, h, w)$ denotes (batch size, input/output channel, height, width), respectively. For encoder $n c=2 * c$ and for decoder $n c=c / / 2$. Right: the structure of the last convolutional combination (conv_last) in decoder plotted as the blue column in Figure 1. 


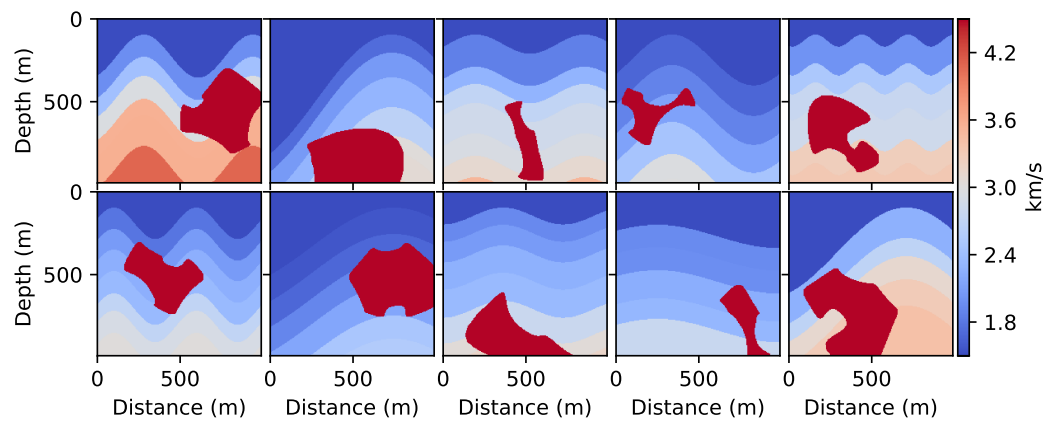

Figure 3: Ten velocity models extracted from the training dataset. 


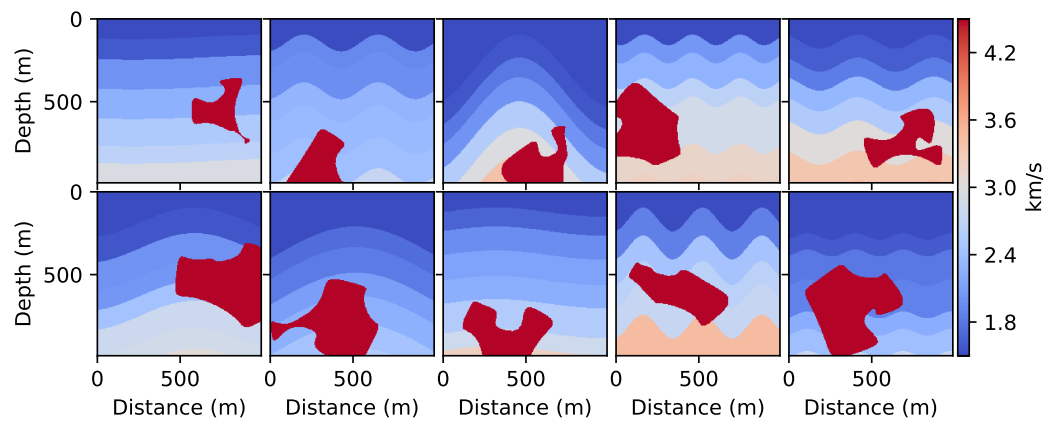

Figure 4: Ten velocity samples selected from the validation dataset. 


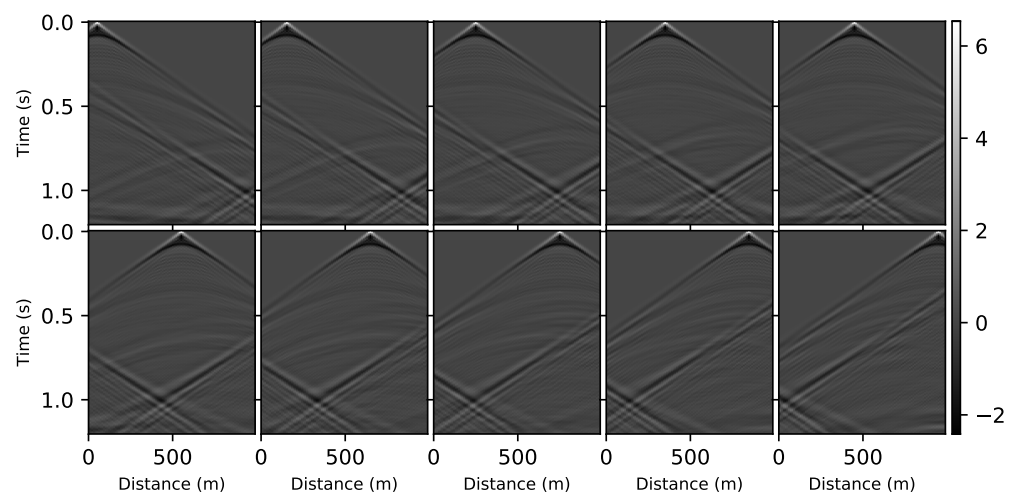

Figure 5: One input example containing ten shot gathers using the velocity model shown in the upper-left corner in Figure 4. 


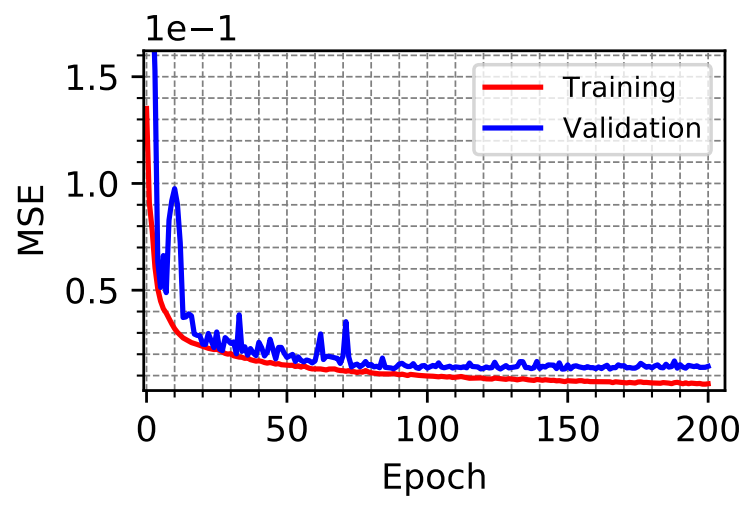

Figure 6: MSE during the training stage using equation 4 as the objective function. 


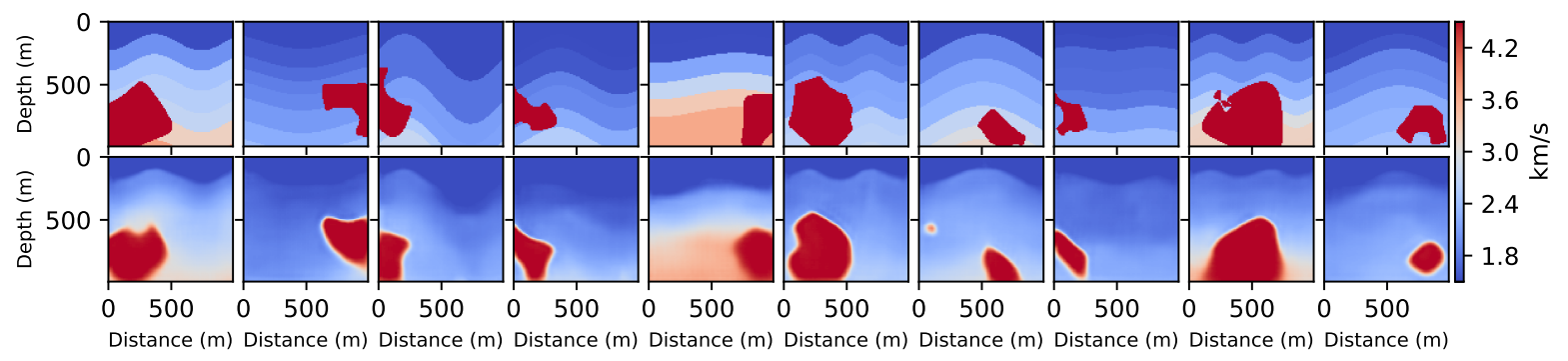

Figure 7: Comparison of the ground-truth (the top row) and the data-driven CNN estimated (the bottom row) velocity models. 


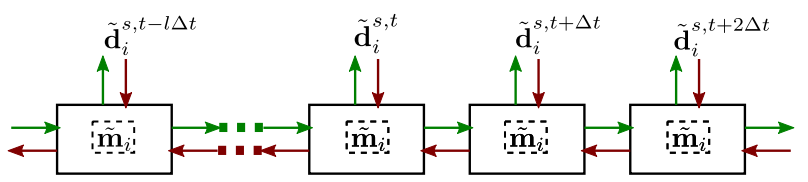

Figure 8: Truncated structure of waveform RNN, where the green (red) arrows delineate the forward (backward) propagation direction. 


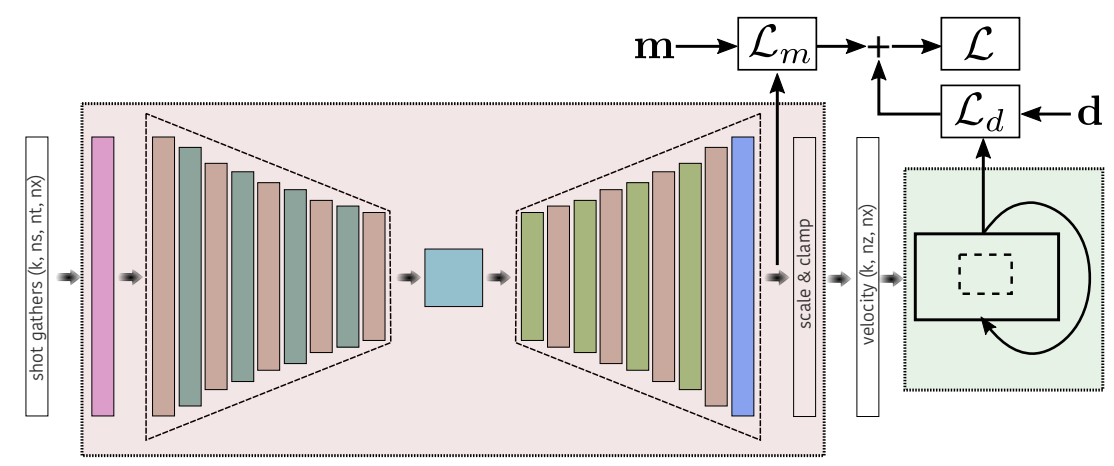

Figure 9: The structure of a physics-guided network involving a data-driven CNN illustrated in Figure 1 and a waveform-based RNN plotted in Figure 8. 


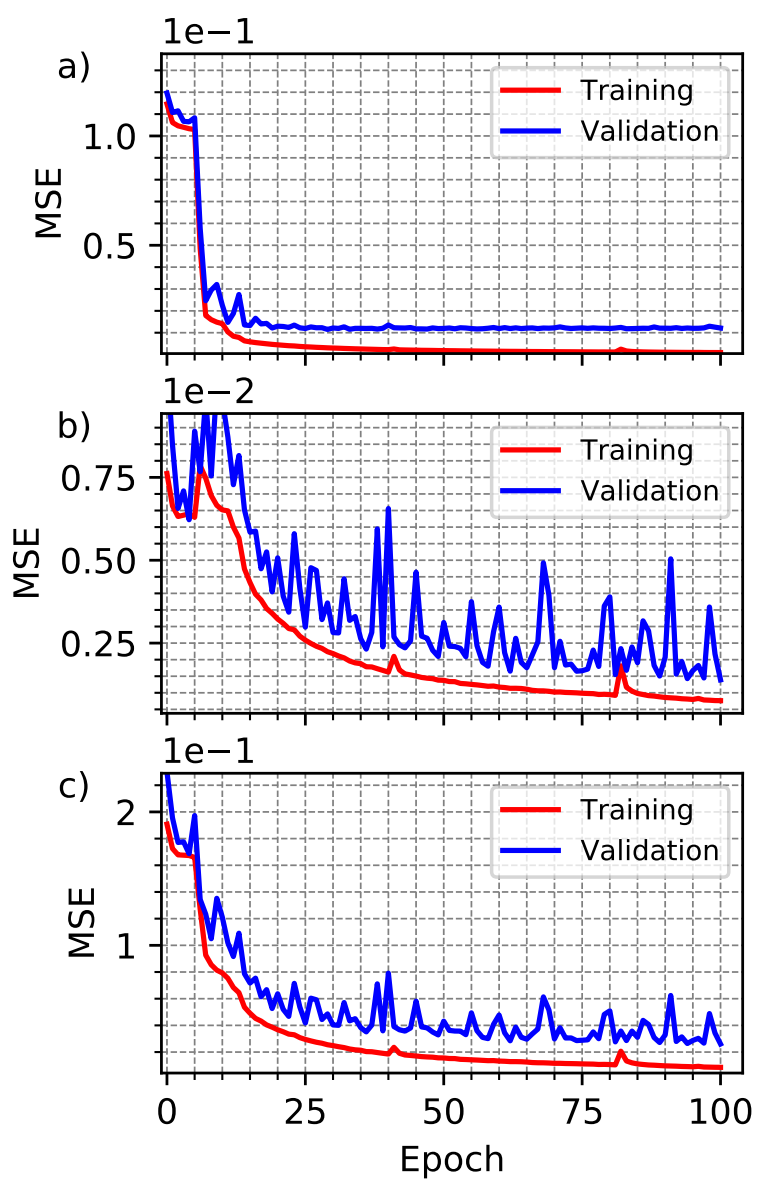

Figure 10: The misfits behavior during a physics-guided training of the network. a) mean squared model misfit $\left.\tilde{\mathcal{L}}_{m}, b\right)$ mean squared data misfit $\left.\tilde{\mathcal{L}}_{d}, c\right)$ weighted summation: $\tilde{\mathcal{L}}_{m}+$ $10 \tilde{\mathcal{L}}_{d}$ 


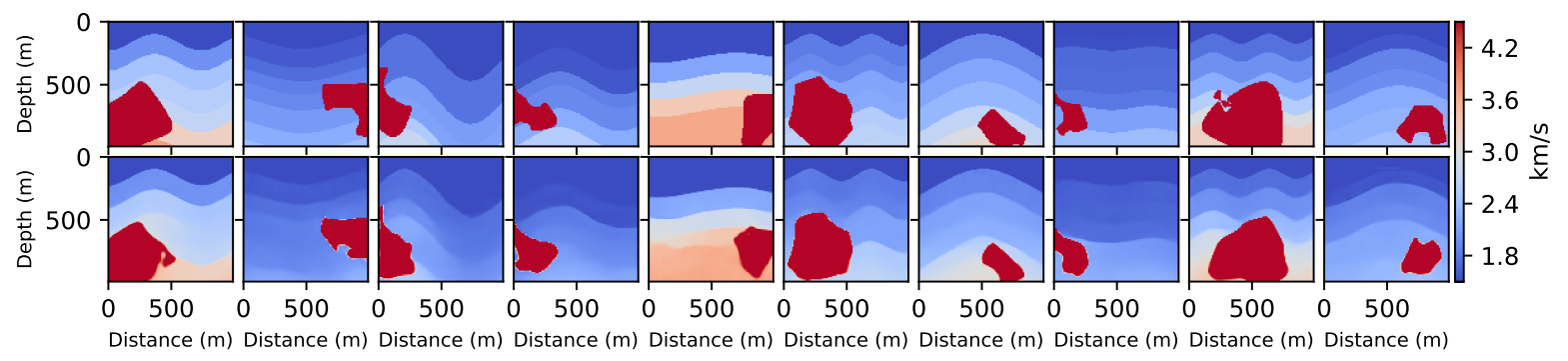

Figure 11: Comparison of the ground-truth velocity models and the physics-guided network estimated results. 


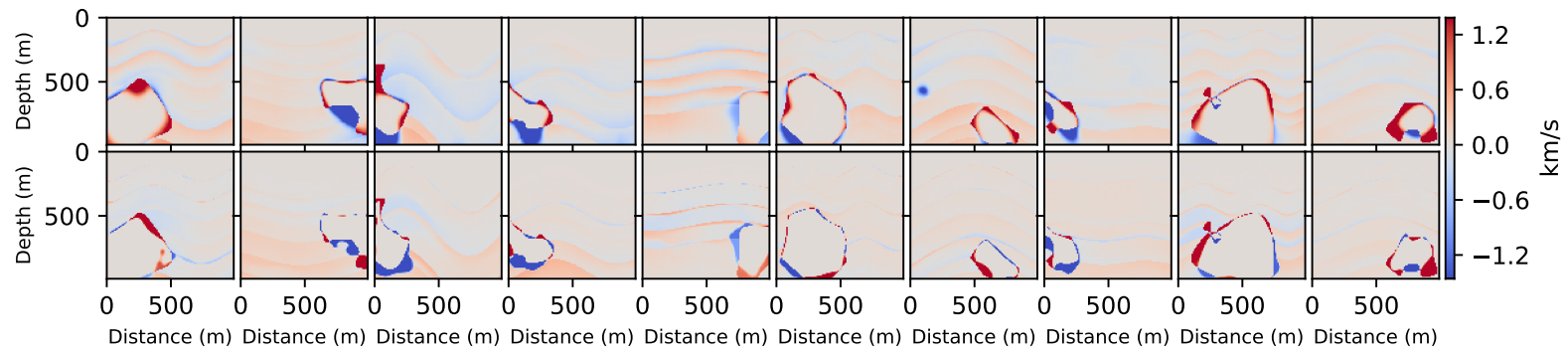

Figure 12: Model misfit comparison between the data-driven CNN and the physics-guided network. 


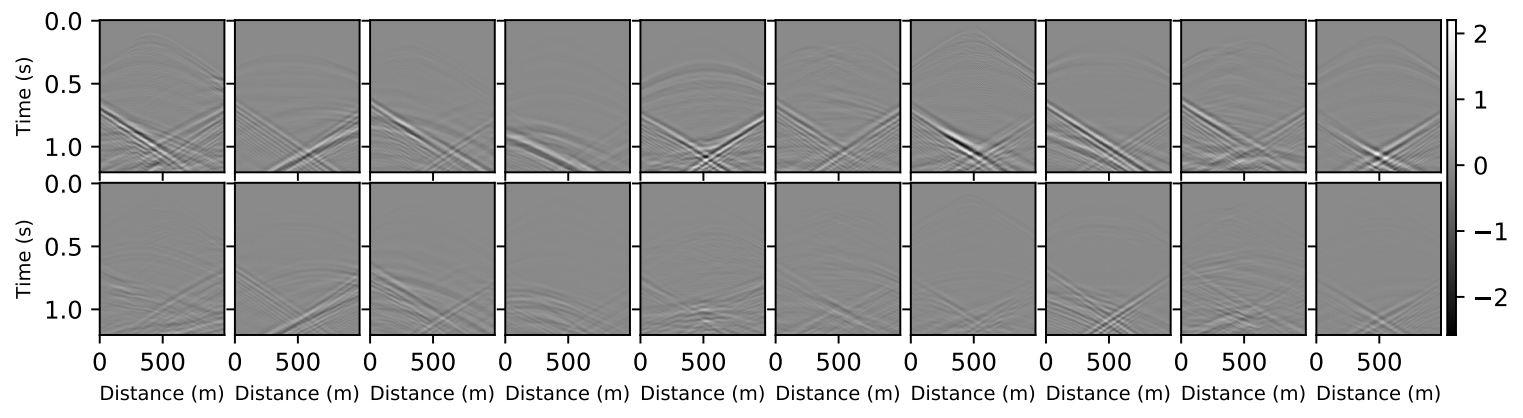

Figure 13: Data residual comparison between the data-driven CNN and the physics-guided network. 


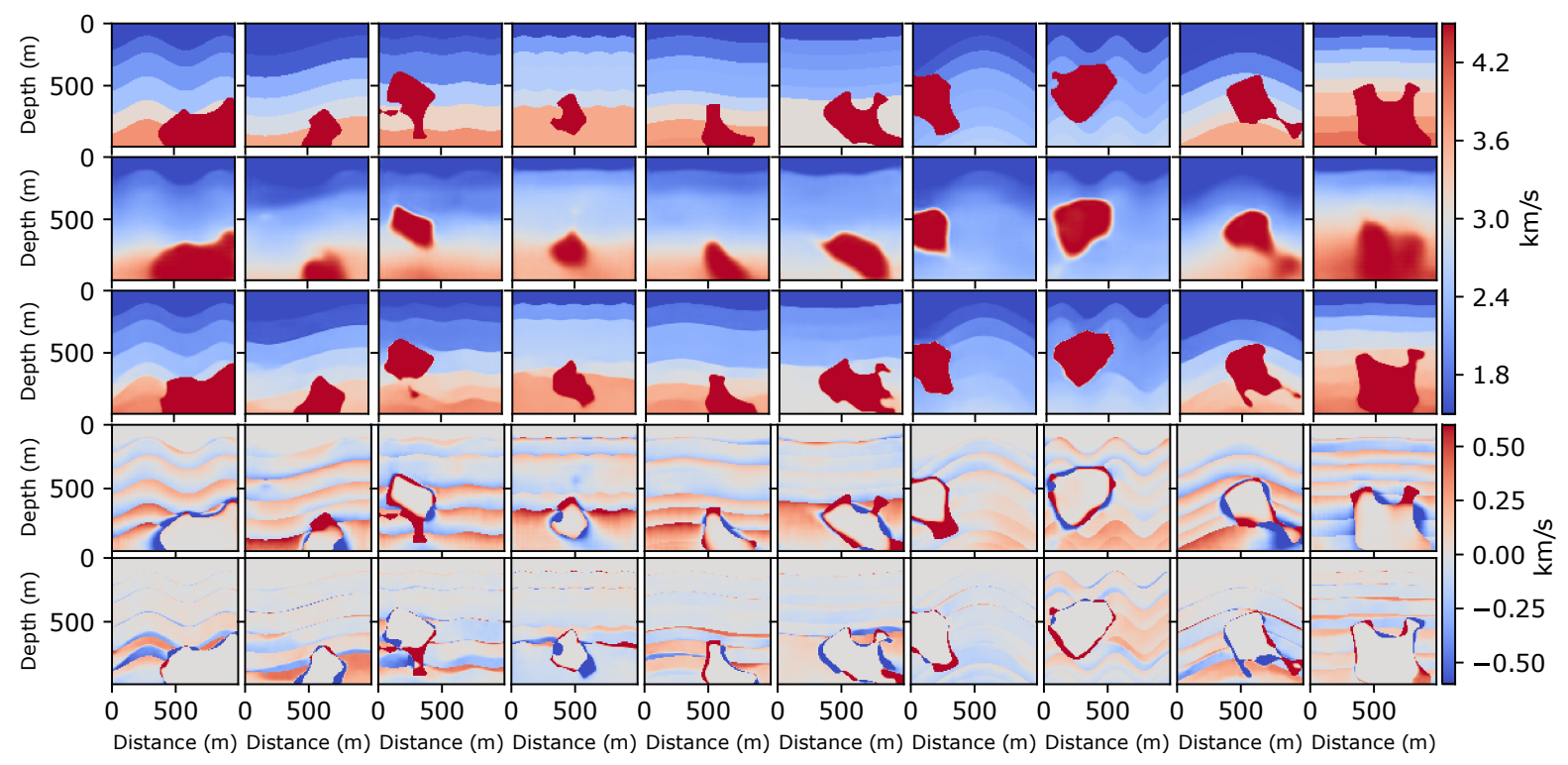

Figure 14: Ten additional velocity samples extracted from the validation dataset. From top to bottom: the ground-truth velocity models, the data-driven estimated results, the physics-guided network results, model misfits from the data-driven $\mathrm{CNN}$, and model misfits from the physics-guided network. 


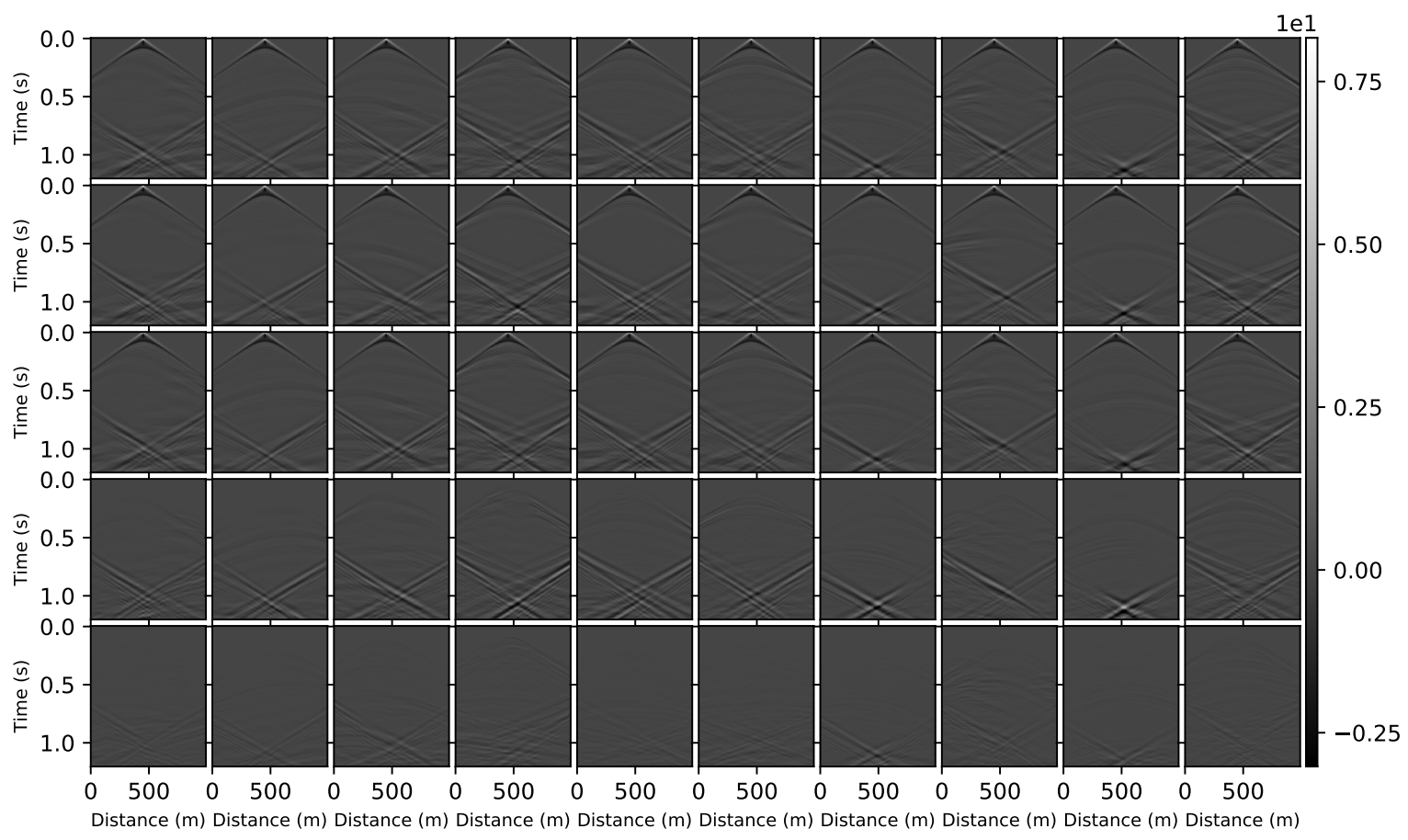

Figure 15: Ten additional middle-located shot gathers and data residuals associated with velocity models, in the same order, illustrated in Figure 14. 


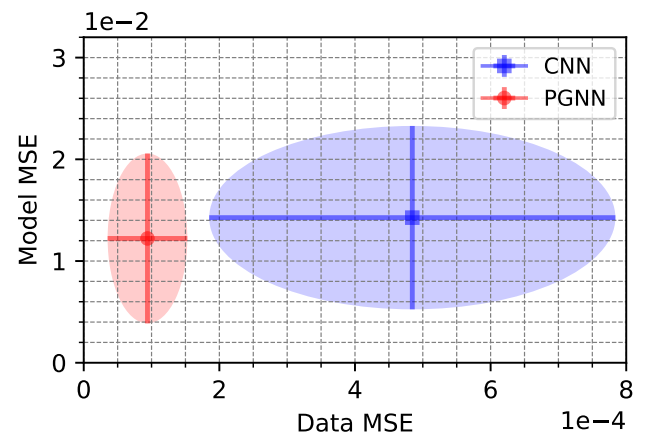

Figure 16: Model and data MSEs analysis between the data-driven CNN and the physicsguided network. 


\section{LIST OF TABLES}

1 Detailed parameters of our proposed CNN with architecture shown in Figure 1. 44 


\begin{tabular}{|c|c|c|c|c|c|c|}
\hline \multicolumn{2}{|c|}{ Operation Layer } & $\begin{array}{l}\text { Number } \\
\text { of Filters }\end{array}$ & $\begin{array}{c}\text { Size of } \\
\text { Each Filter }\end{array}$ & Stride & Padding & $\begin{array}{l}\text { Output Size } \\
(\text { nc } x \mathrm{~h} \text { x } \mathrm{w})\end{array}$ \\
\hline Input Data & shot gathers & - & - & - & - & $10 \times 400 \times 100$ \\
\hline Initial Convolution & conv_init & 16 & $1 \times 1 \times 10$ & $1 \mathrm{x} 1$ & 0 & $16 \times 400 \times 100$ \\
\hline \multirow{14}{*}{ Encoder } & \multirow{2}{*}{ conv_block } & 32 & $3 \times 3 \times 16$ & $1 \mathrm{x} 1$ & $1 \mathrm{x} 1$ & $32 \times 400 \times 100$ \\
\hline & & 32 & $3 \times 3 \times 32$ & $1 \mathrm{x} 1$ & $1 \mathrm{x} 1$ & $32 \times 400 \times 100$ \\
\hline & max_pool & 1 & $4 \times 2$ & $4 \times 2$ & $2 \mathrm{x} 0$ & $32 \times 101 \times 50$ \\
\hline & \multirow{2}{*}{ conv_block } & 64 & $3 \times 3 \times 32$ & $1 \mathrm{x} 1$ & $1 \mathrm{x} 1$ & $64 \times 101 \times 50$ \\
\hline & & 64 & $3 \times 3 \times 64$ & $1 \mathrm{x} 1$ & $1 \mathrm{x} 1$ & $64 \times 101 \times 50$ \\
\hline & max_pool & 1 & $3 \times 2$ & $2 \times 2$ & $1 \mathrm{x} 0$ & $64 \times 51 \times 25$ \\
\hline & \multirow{2}{*}{ conv_block } & 128 & $3 \times 3 \times 64$ & $1 \mathrm{x} 1$ & $1 \mathrm{x} 1$ & $128 \times 51 \times 25$ \\
\hline & & 128 & $3 \times 3 \times 64$ & $1 \mathrm{x} 1$ & $1 \mathrm{x} 1$ & $128 \times 51 \times 25$ \\
\hline & max_pool & 1 & $4 \times 2$ & $4 \times 2$ & $0 \mathrm{x} 0$ & $128 \times 12 \times 12$ \\
\hline & \multirow{2}{*}{ conv_block } & 256 & $3 \times 3 \times 128$ & $1 \mathrm{x} 1$ & $1 \mathrm{x} 1$ & $256 \times 12 \times 12$ \\
\hline & & 256 & $3 \times 3 \times 128$ & $1 \mathrm{x} 1$ & $1 \mathrm{x} 1$ & $256 \times 12 \times 12$ \\
\hline & max_pool & 1 & $2 \times 2$ & $2 \times 2$ & $0 \mathrm{x} 0$ & $256 \times 6 \times 6$ \\
\hline & \multirow{2}{*}{ conv_block } & 512 & $3 \times 3 \times 256$ & $1 \mathrm{x} 1$ & $1 \mathrm{x} 1$ & $512 \times 6 \times 6$ \\
\hline & & 512 & $3 \times 3 \times 512$ & $1 \mathrm{x} 1$ & $1 \mathrm{x} 1$ & $512 \times 6 \times 6$ \\
\hline \multirow{13}{*}{ Decoder } & conv_trans & 512 & $2 \times 2 \times 512$ & $2 \times 2$ & $0 \mathrm{x} 0$ & $512 \times 12 \times 12$ \\
\hline & \multirow{2}{*}{ conv_block } & 256 & $3 \times 3 \times 512$ & $1 \mathrm{x} 1$ & $1 \mathrm{x} 1$ & $256 \times 12 \times 12$ \\
\hline & & 256 & $3 \times 3 \times 256$ & $1 \mathrm{x} 1$ & $1 \mathrm{x} 1$ & $256 \times 12 \times 12$ \\
\hline & conv_trans & 256 & $3 \times 3 \times 256$ & $2 \times 2$ & $0 \mathrm{x} 0$ & $256 \times 25 \times 25$ \\
\hline & \multirow{2}{*}{ conv_block } & 128 & $3 \times 3 \times 256$ & $1 \mathrm{x} 1$ & $1 \mathrm{x} 1$ & $128 \times 25 \times 25$ \\
\hline & & 128 & $3 \times 3 \times 128$ & $1 \mathrm{x} 1$ & $1 \mathrm{x} 1$ & $128 \times 25 \times 25$ \\
\hline & conv_trans & 128 & $2 \times 2 \times 128$ & $2 \times 2$ & $0 \mathrm{x} 0$ & $128 \times 50 \times 50$ \\
\hline & \multirow{2}{*}{ conv_block } & 64 & $3 \times 3 \times 128$ & $1 \mathrm{x} 1$ & $1 \mathrm{x} 1$ & $64 \times 50 \times 50$ \\
\hline & & 64 & $3 \times 3 \times 64$ & $1 \mathrm{x} 1$ & $1 \mathrm{x} 1$ & $64 \times 50 \times 50$ \\
\hline & conv_trans & 64 & $2 \times 2 \times 64$ & $2 \times 2$ & $0 \mathrm{x} 0$ & $64 \times 100 \times 100$ \\
\hline & \multirow{2}{*}{ conv_block } & 32 & $3 \times 3 \times 64$ & $1 \mathrm{x} 1$ & $1 \mathrm{x} 1$ & $32 \times 100 \times 100$ \\
\hline & & 32 & $3 \times 3 \times 32$ & $1 \mathrm{x} 1$ & $1 \mathrm{x} 1$ & $32 \times 100 \times 100$ \\
\hline & conv_last & 1 & $1 \times 1 \times 32$ & $1 \mathrm{x} 1$ & $0 \mathrm{x} 0$ & $1 \times 100 \times 100$ \\
\hline \multirow{2}{*}{ scale \& clamp } & scale & \multicolumn{5}{|c|}{$\tilde{\mathbf{m}}=\tilde{\mathbf{m}} *\left(\mathbf{m}_{\max }-\mathbf{m}_{\min }\right)+\mathbf{m}_{\min }$} \\
\hline & clamp & \multicolumn{5}{|c|}{$\tilde{\mathbf{m}}= \begin{cases}\mathbf{m}_{\min } & \text { if } \tilde{\mathbf{m}} \leq \mathbf{m}_{\min } \\
\tilde{\mathbf{m}} & \text { otherwise } \\
\mathbf{m}_{\max } & \text { if } \tilde{\mathbf{m}} \geq \mathbf{m}_{\max }\end{cases}$} \\
\hline
\end{tabular}

Table 1: Detailed parameters of our proposed CNN with architecture shown in Figure 1. 